\title{
PERFORMANCE EVALUATION OF THE METHODS USED FOR THE DETERMINATION OF SULFUR CONTENT IN FUELS AND BIOFUELS
}

\author{
$\underline{\text { Ileana Nicolescu}}{ }^{1}$, Agnes Serbanescu $^{1}$, Mona Barbu$^{1}$, Elena Bucur ${ }^{1}$, Raluca Diodiu ${ }^{1}$ \\ 1 National Research \& Development Institute for Industrial Ecology - ECOIND, 90-92 \\ Panduri Str., Bucharest, ileana.nicolescu@incdecoind.ro, Romania
}

\begin{abstract}
This paper presents the results of an experimental research study having as objective the development of a routine method based on ion - chromatography technique, used for the sulfur content determination in solid and liquid fuels.

The proposed procedure was applied on the determination of total sulfur in samples of coal, fuel oil and biomass with different sulfur content ranging from $0.05 \%$ to $0.6 \%$.

The methodology consists of the sample preparation by Eschka method based on igniting a test portion of coal sample in intimate contact with Eschka mixture at $800^{\circ} \mathrm{C}$, or by combustion of the fuel oil or biomass sample in a closed system containing oxygen (calorimeter bomb), followed by the selective determinations of sulfur content using ion-chromatography and gravimetric techniques.

When ion chromatography technique is used for sulfur content determination in coal and fuel oil, the precision of values expressed as the relative standard deviations (\% RSD, $n=10$ ), were $1.47 \%$ and $0.61 \%$, which has been understood as being much lower than those obtained by gravimetric standard methods, more precisely, $3.46 \%$ for coals and $2.46 \%$ for fuel oil.

For biomass with a sulfur content about 10 times less than in coals and fuel oil, best results were obtained by ion - chromatography technique with the low limit of the measuring range much smaller than the limit reached by applying the gravimetric technique.
\end{abstract}

Keywords: Ion Chromatography, Fuels, Gravimetric method, Sulfur, Eschka mixture

\section{Introduction}

Romania has significant energy resources, including oil, coal and natural gas, and has a long coal mining tradition, stretching back 150 years.

The transposed EU laws on environmental protection oblige the coal industry to meet several European standards.

Thus, 2001 / 80 / EC Directive on Large Combustion Plants (LCP Directive) was adopted and implemented. The overall aim of the LCP Directive is to reduce emissions of acidifying pollutants, particles, and ozone precursors. Control of emissions from large combustion plants - those whose rated thermal input is equal to or greater than $50 \mathrm{MW}$ - plays an important role in the Union's efforts to combat acidification, eutrophication and ground-level ozone as part of the overall strategy to reduce air pollution.

Regarding the biomass, it is used in Romania, mostly for thermal energy generation. Studies on the consumption of biomass in our country shows that $50 \%$ from the generated heat had as source forest biomass burning, being obviously that the biomass plays and will play an important role in the Romanian energetic sector. [1] Biomass fuels have negligible sulfur content and, therefore, do not contribute to sulfur dioxide emissions as the classic fuels [2]. Even so, in accordance with EN ISO 17225$2: 2014$, sulfur content in biomass is limited for industrial purposes at no more than 0.05 $\%$. 
With national and international regulations, as well as traded fuel specifications increasingly stringent, the determination of sulfur content became a critical value in quantifying fuels like coals, petroleum oils, fuel products biomass and other alternative fuels and therefore, an accurately measuring and monitoring sulfur is of prime importance.

Considering all the above, the present work aims to develop an ion chromatographic method for the determination of total sulfur content in petroleum products and solid fuels (coal, coke and solid biomass).

In parallel with ion -chromatographic technique, the gravimetric method was used as reference for both liquid and solid fuels.

\section{Experimental}

\subsection{Standard samples containing sulfur}

Two commercially available $1000 \mathrm{mg} / \mathrm{L}$ certified solution of sulfate (CertiPUR, Merck and Sulfate Standard for IC TraceCERT®, Sigma-Aldrich), were used to prepare the calibration curve for ion chromatography method and to verify the accuracy of the sulfur determination as sulfate ion.

\subsection{Samples}

The methods were tested on samples of coal and fuel oil with sulfur content of 0.401 $\%$ and $0.493 \%$ respectively, received from the providers of PT schemes.

For lower levels of sulfur, an in - house sample with sulfur content of $0.2 \%$ (cellulose and sulphanilic acid) and a sample of solid biomass (sawdust) with sulfur content of $0.04 \%$ were used.

Six determinations per sample were performed.

\subsection{Reagents}

All reagents were prepared using ultrapure water $(<0.1 \mu \mathrm{S} / \mathrm{cm})$ obtained from a purification system (Ultra Clear TWF UV Siemens SG Water, Germany), and all chemicals used were of analytical quality.

2.3.1 $30 \mathrm{mM}$ Sodium Hydroxide eluent solution for lon chromatography method. [3]

2.3.2 Potassium sulfate, dried at $105^{\circ} \mathrm{C}$, was dissolved in water to prepare $2 \mathrm{~g} / \mathrm{L}$ solution that was used for blank test in order to verify a possible contamination or loss of sulfate ion.

2.3.3 Hydrochloric acid, $1.18 \mathrm{~g} / \mathrm{ml}$ (Merck, Darmstadt, Germany), for the dissolution of the residue.

2.3.4 Barium chloride, $85 \mathrm{~g} / \mathrm{L}$, to precipitate the sulfate ion.

2.3.5 Methyl red indicator solution.

2.3.6 Ammonia, $25 \%$;

2.3.7 Silver nitrate to verify the presence of chlorides in washing waters of the barium sulfate precipitate. [4]

2.3.8 Hydrogen peroxide, $30 \%$ to oxidize the resulting sulfur dioxide in calorimeter bomb, to sulfate anion.

The reagents 2.3.2 - 2.3.7 were used for gravimetric Eschka method and 2.3.8 for sample preparation with calorimeter bomb. 


\subsection{Methods}

\subsubsection{Particle size-reduction of the solid fuels}

Solid samples (coals and biomass) were prepared in accordance with EN ISO 52642:2011 and EN 14780:2011. The samples were milled and sieved to $200 \mu \mathrm{m}$ after airdried, sealed in plastic bottles, and stored at $20^{\circ} \mathrm{C}$.

In this purpose, a Rotor Beater Mill SR 300 for coals and a Vibratory Disc Mill RS 200 - Retsch for biomass, were used. (Figures 1 and 2)

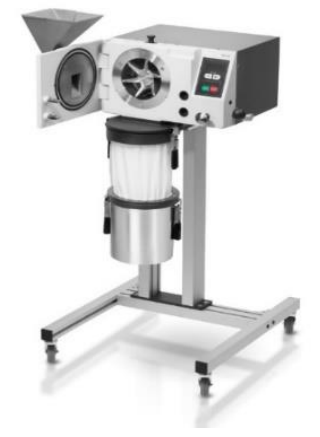

Figure 1 - Rotor Beater Mill SR 300

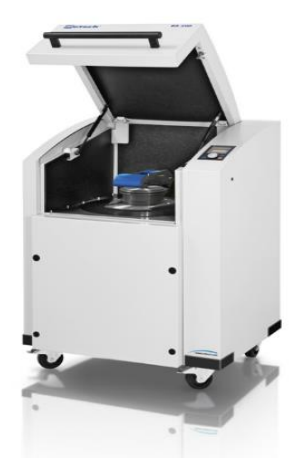

Figure 2 - Vibratory Disc Mill RS 200

2.4.2 Fuel sample preparation for lon - Chromatography and Gravimetric determinations

The sample of coal was prepared in accordance with the procedure described in ISO 334 , when a mass of about $1 \mathrm{~g}$ of coal was ignited at $800^{\circ} \mathrm{C}$ in intimate contact with Eschka mixture in an oxidizing atmosphere to remove combustible matter and to convert sulfur in sulfate. Subsequent, the sulfate ion was extracted by washing the residues with ultrapure water. The resulted solution was diluted to $250 \mathrm{~mL}$ volumetric flask and the sulfate anion was determined by ion chromatography technique.

In other case, Eschka residue was dissolved with hydrochloric acid solution and then, the sulfate anion was gravimetrically determined by precipitation with barium chloride. EN ISO 16994:2015 [5] and ASTM D 129:2013 [6] use the decomposition of biomass and fuel oil in bomb calorimeter at $30 \mathrm{~atm}$ and the presence of oxygen, and the resulting sulfur dioxide is oxidized with hydrogen peroxide to sulfate which is subsequently determined by ion chromatography, after an appropriate dilution to 100 $\mathrm{mL}$ volumetric flask in the case of biomass and to $250 \mathrm{ml}$ in the case of in - house sample and fuel oil, or gravimetrically by precipitation with barium chloride.

The weighed mass of biomass and in - house sample was about $1 \mathrm{~g}$ and those for fuel oil was about $0.7 \mathrm{~g}$.

\subsubsection{Ion Chromatography method}

Instrumentation

A Dionex ICS-5000 + ion chromatography system (Thermo Scientific, Sunnyvale, CA, USA) was used for the experiments. 
The system is equipped with a conductivity detector, an $\operatorname{lonPac}{ }^{\circledR}$ AS18 hydroxide selective anion-exchange column $(2 \mathrm{~mm})$, operating at a flow rate of $0.25 \mathrm{~mL} / \mathrm{min}$, sample volume $5 \mu \mathrm{L}$ and incorporates an electrolytic eluent generator (for $30 \mathrm{mM}$ $\mathrm{NaOH}$ production), dual-piston pump with vacuum degas, two six-port injection valves fitted with two loops in series set at $5 \mu \mathrm{L}$ ), and suppressor AS 500, $2 \mathrm{~mm}$.

A Thermo Scientific Dionex AS DV Autosampler was used for sample processing.

\section{Calibration curve}

The concentrations of sulfur as sulfate in the solutions corresponding to the sample of biomass were about $5 \mathrm{mg} / \mathrm{L}$ and those corresponding to the samples of coal, fuel oil and in-house sample, were about $50 \mathrm{mg} / \mathrm{L}$, for which reason, were built two calibration curves in the range of $2.5 \mathrm{mg} / \mathrm{L}$ up to $20 \mathrm{mg} / \mathrm{L}$ and from $10 \mathrm{mg} / \mathrm{L}$ to $250 \mathrm{mg} / \mathrm{L}$.

In order to check the calibration curve, were used reference materials of which levels of sulfate were similar to those obtained by preparation of the real samples.

Thus, 6 standards, for each calibration curve were prepared in $25 \mathrm{~mL}$ volumetric flasks by a proper dilution of the certified solution of sulfate (CertiPUR), and also, 3 control samples of each $50 \mathrm{mg} / \mathrm{L}$ and $5 \mathrm{mg} / \mathrm{L}$, respectively were prepared from Sulfate Standard for IC TraceCERT®.

Ion Retention times for Sulfate was 5.3 min.

\subsubsection{Gravimetric method}

Sulfur solutions obtained by sample preparations of coals, fuel oil, biomass and in house - samples as described at 2.4.2, were further processed in accordance with 7.6 - 7.7 and 6.5 - 6.6 paragraphs of ISO 334: 2013 and ASTM D 129-136.5, respectively, in which are described the procedures for sulfate ion precipitation with barium chloride, followed by the ignition of the sample with barium sulfate content.

\section{Results and Discussion}

3.1. Analysis of standard samples using Dionex ICS-5000 + ion chromatography system

\subsubsection{Linearity}

The analytical curves, $Y=a+b C$, were constructed by plotting the area of the chromatographic peak of the sulfate ion, $Y(\mu S * \mathrm{~min})$, versus the concentration, $C$, of the analyte.

The experimental coefficients of the linear regressions: $2.5 \mathrm{mg} / \mathrm{L}-20 \mathrm{mg} / \mathrm{L}$ and 10 $\mathrm{mg} / \mathrm{L}-250 \mathrm{mg} / \mathrm{L}$ are: $\mathrm{R}=0.9999$ and $\mathrm{R}=0.9998$, respectively.

Calibration curves and Chromatogram of the standard solution, are shown in figures 1 and 2. 


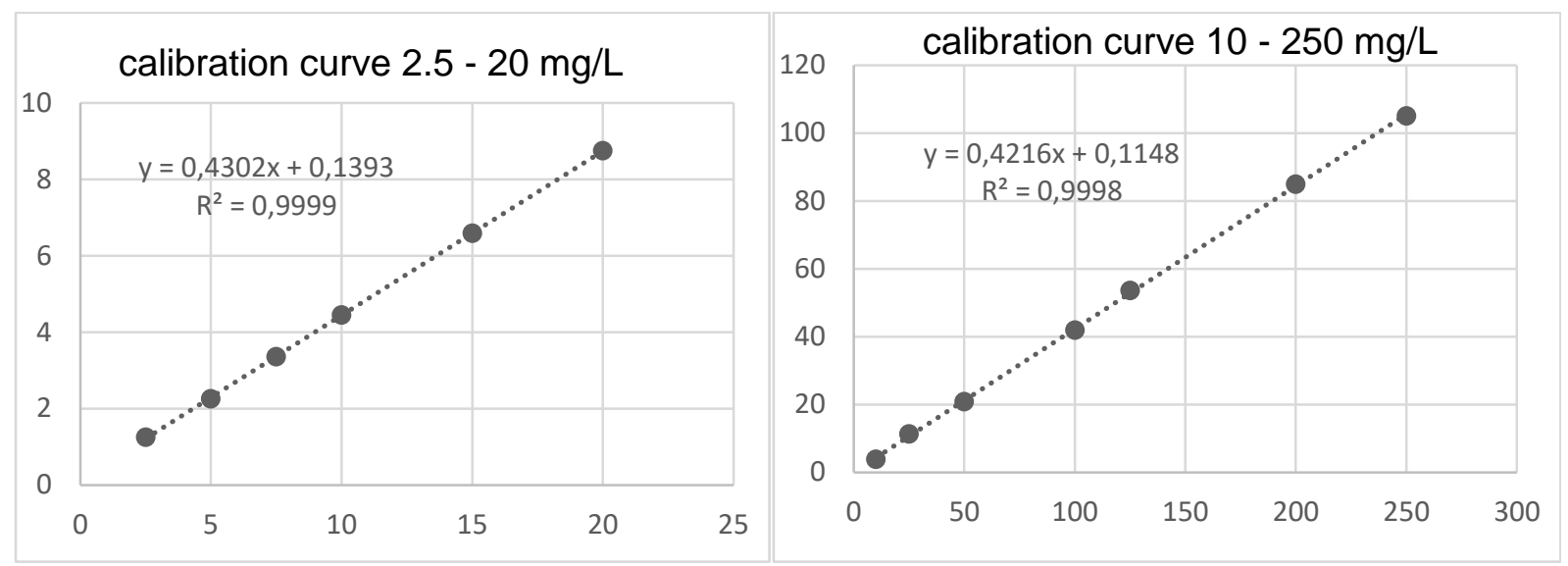

Figure 1. Calibration curves of standard solutions for sulfate anion

$\mathrm{s}$

Figure 2 Chromatogram of the standard solution of sulfate anion

The limits of detection (LOD) and quantitation (LOQ) were calculated considering LOD $=3 S$ and $L O Q=10 S$, where $S$ is the standard deviation determined by repeated injections of blank, which in our case, is ultrapure water used for dilution of the standard solutions.[7]. The obtained results are shown in Table 1.

Table 1 - The analytical parameters obtained for the ion - chromatograph determination of sulfate anion using standard solutions

\begin{tabular}{ccccccc}
$\begin{array}{c}\text { Measuring } \\
\text { range }\end{array}$ & $\begin{array}{c}\text { Retention } \\
\text { time }\end{array}$ & \multicolumn{2}{c}{ Analytical equation } & $\mathbf{R}$ & LOD & LOQ \\
{$[\mathbf{m g} / \mathbf{L}]$} & {$[\mathbf{m i n}]$} & $\mathbf{a}$ & $\mathbf{b}$ & & {$[\mathbf{m g} / \mathbf{L}]$} & {$[\mathbf{m g} / \mathbf{L}]$} \\
$2.5-20$ & & 0.014 & 0.43 & 0.9999 & 0.023 & 0.071 \\
$10-$ & 5.3 & 0.011 & 0.42 & 0.9998 & 0.039 & 0.116 \\
250 & & & & &
\end{tabular}

The limits of detection and quantitation obtained for the proposed method were: LOD - $0.023 \mathrm{mg} / \mathrm{L}$ and LOQ - $0.071 \mathrm{mg} / \mathrm{L} ; \mathrm{LOD}-0.039 \mathrm{mg} / \mathrm{L}$ and LOQ $-0.116 \mathrm{mg} / \mathrm{L}$ for the lower and higher levels of sulfate ion, respectively.

\subsubsection{Precision}

The precision was evaluated according to the repeatability and was expressed as relative standard deviation (RSD). Two chromatographic parameters, the retention time and the area of the analytical signal were studied to evaluate the repeatability. In 
this goal, two standard solutions containing the sulfate, $5.0 \mathrm{mg} / \mathrm{L}$ and $50 \mathrm{mg} / \mathrm{l}$ anions were analyzed six times by consecutive injections into the ion-chromatograph. The mean values obtained for the retention time and the area of the signal as well as their RSD values are shown in Table 2.

Table 2 Repeatabilities of the retention time and the analytical signal for the sulfate ion

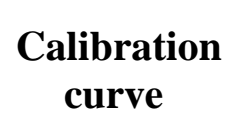

$[\mathrm{mg} / \mathrm{L}]$

$2.5-20$

$10-250$

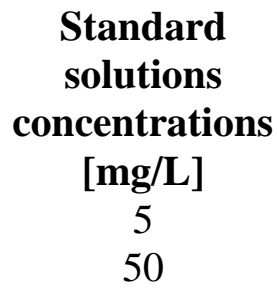

$\begin{gathered}\text { Retention } \\ \text { time }\end{gathered}$
[min]
5.3

RSD

[\%]

0.06

0.05

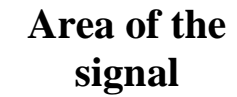

RSD

$\begin{array}{cc}{\left[\mu \mathbf{S ~ c m}^{-1} \mathbf{~ m i n}\right]} & {[\%]} \\ 2.625 & 0.21 \\ 22.021 & 0.17\end{array}$

It can be concluded that the method presents good repeatability because in both cases the observed RSD was below $1 \%$.

\subsubsection{Accuracy}

The accuracy of the method was evaluated through recovery tests.

In this regard were used the sulfur solutions with different levels of sulfur as sulfate content of about $50 \mathrm{mg} / \mathrm{L}$ and $5 \mathrm{mg} / \mathrm{L}$, obtained by sample preparation of coal, fuel oil biomass and in - house sample. Therefore, both calibration curves were evaluated. The results in $\mathrm{mg} \mathrm{SO}_{4} / \mathrm{L}$ were then recalculated to obtain sulfur content in initial samples as mass percent.

The resuts obtained by ion - chromatography were compared with those obtained in parallel by standard gravimetric method.

For each sample were performed 10 determinations. The results of the recovery are shown in Table 3.

Table 3 Results of the recovery of the sulfur in fuels

\begin{tabular}{|c|c|c|c|c|c|c|c|c|c|}
\hline \multirow[t]{3}{*}{ Sample } & \multicolumn{3}{|c|}{ Concentration, [\%] } & \multicolumn{2}{|c|}{ Recovery, [\%] } & \multicolumn{2}{|c|}{$\mathrm{SD},[\%]$} & \multicolumn{2}{|c|}{ RSD, [\%] } \\
\hline & Initial & IL & Gravim & IC & Gravim & IC & Gravim & IC & Gravir \\
\hline & & & & & & & & & \\
\hline & 0.401 & 0.407 & 0.404 & 102.5 & 104.0 & 0.006 & 0.014 & 1.47 & 3.46 \\
\hline uel o & 0.493 & 0.488 & 0.4 & 98.99 & & 0.003 & & 0.61 & 2.46 \\
\hline & 0 & & & & & & & 1.6 & 2.82 \\
\hline Biomass & 0.040 & 0.038 & 0.043 & 95.00 & 107.5 & 0.002 & 0.004 & 5.00 & 10.0 \\
\hline
\end{tabular}

In according with ISO $334: 2013$ and D 129:2013, standard deviation is 0.018 for coals and 0.014 for petroleum products. Standard deviations obtained by ion chromatography was 0.006 for coal and and 0.003 for fuel oil, these values being much lower than those provided by standards.

The recoveries varied from 95.0 to $104 \%$ and from 100.4 to 107.5 for ion chromatography and gravimetric methods, respectively, indicating that the extraction of the analytes and the techniques applied are properly to be used for the determination of the sulfate anion in liquid and solid fuels, and also for solid biomass.

Currently, in analytical procedures, recovery percentages in the range of $80-120 \%$ with a precision of approximately $20 \%$ are acceptable. [8] 


\section{Conclusions}

In this paper was proposed an ion - chromatographic method to determine the sulfur content in fuels and preliminary results are presented.

First stage of the experiments consisted in the development of the ion chromatographic method to measure the sulfate ion in aqueous solutions and to establish the performance parameters using certified reference materials.

As the sulfur solutions from real samples preparation, had different levels of sulfate anion ( $5 \mathrm{mg} / \mathrm{L}$ and $50 \mathrm{mg} / \mathrm{L}$ ) it was decided to use 2 measuring ranges as follow: 2.5 $\mathrm{mg} / \mathrm{L}$ up to $20 \mathrm{mg} / \mathrm{L}$ and $10 \mathrm{mg} / \mathrm{L}$ to $250 \mathrm{mg} / \mathrm{L}$.

The limits of detection and quantitation obtained for the proposed method in were: LOD - $0.023 \mathrm{mg} / \mathrm{L}$ and LOQ - $0.071 \mathrm{mg} / \mathrm{L} ; \mathrm{LOD}-0.039 \mathrm{mg} / \mathrm{L}$ and LOQ $-0.116 \mathrm{mg} / \mathrm{L}$ for the lower and higher levels of sulfate ion, respectively.

Repeatability tests with reference materials, expressed as relative standard deviation (RSD), were $0.21 \%$ and $0.17 \%$, showing a good precision of the method.

The accuracy of the method was evaluated through recovery tests, using sulfur solutions obtained from real samples of coals, fuel oils, biomass and an in - house sample.

Samples preparation was performed by Eschka method for coals and bomb calorimeter method for biomass, fuel oil and in -house sample.

Standard deviations obtained by ion - chromatography was 0.006 for coal and and 0.003 for fuel oil, these values being much lower than those provided by the standards in force.

The recoveries varied from 95.0 to $104 \%$ and from 100.4 to 107.5 for ion chromatography and gravimetric methods, respectively, indicating that the extraction of the analytes and the techniques applied are properly to be used for the determination of the sulfate anion in liquid and solid fuels, and also for solid biomass.

Considering all the above, it is obviously that for the new ion - chromatographic method developed in laboratory, the performance parameters obtained and the recovery tests are in accordance with the standardized gravimetric methods and are suitable to measure the sulfur content as sulfate anion in different fuels.

For solid samples as sawdust with low sulfur content (10 times less than in coals and fuel oil), best results were obtained by ion - chromatography technique with the low limit of the measuring range much smaller than the limit reached by applying the gravimetric technique.

\section{References}

[1] Dr. Stelian A. Borz, Dr. Rudolf Derczeni, Dr. Bogdan Popa, Dr. Mihai-Daniel Nita, (2013), Regional Profile of the Biomass Sector in Romania, Foropa - Biomass to the masses, Brasov, Romania;

[2] Camelia Ciubota - Rosie*, Maria Gavrilescu, Matei Macoveanu, (September/October 2008), Biomass - An important renewable source of energy in romania, Environmental Engineering and Management Journal, Vol.7, No.5, 559-568;

[3] BS EN ISO 10304-1:2009 Water quality. Determination of dissolved anions by liquid chromatography of ions. Determination of bromide, chloride, fluoride, nitrate, nitrite, phosphate and sulfate

[4] ISO $334-2013$ Solid mineral fuels - Determination of total sulfur - Eschka method

[5] SR EN ISO 16994:2015 Solid biofuels - Determination of total content of sulfur and chlorine; 
[6] ASTM D 129 - 13 - Standard Test Method for Sulfur in Petroleum Products (General High Pressure Decomposition Device Method)

[7] Eva Lúcia Cardoso Silveira, Lilia Basílio de Caland, Matthieu Tubino, (2014), Simultaneous quantitative analysis of the acetate, formate, chloride, phosphate and sulfate anions in biodiesel by ion chromatography, Elsevier Journal, Vol. 124, 97 - 101;

[8] EPA METHOD 300 - Determination of inorganic anions by ion chromatography, John D. Pfaff Inorganic Chemistry Branch Chemistry Research Division, 